\title{
AVALIAÇÃO DE PROGRAMAS PREVENTIVOS: RELATO DE EXPERIÊNCIA
}

\author{
Suzane Schmidlin Löhr \\ Ana Carina Stelko Pereira \\ André Luiz Monezi Andrade \\ Luziane de Fátima Kirchner"I
}

\begin{abstract}
RESUMO. A análise criteriosa de um projeto permite refiná-lo identificando seus aspectos dificultadores e facilitando a inclusão de estratégias efetivas para correção dos problemas. Alguns autores sugerem que a avaliação de programas preventivos seja feita considerando-se as etapas: análise do problema, delineamento do programa, realização de teste-piloto, realização de teste avançado, disseminação ou ampliação para outros contextos. O presente artigo, observando as referidas etapas, procurou avaliar um projeto preventivo que visa a estimular o desenvolvimento de habilidades sociais em crianças de 6 a 8 anos. Constatou-se que o projeto analisado tem vários aspectos positivos: promove mudanças sutis no comportamento das crianças e sensibiliza os pais para que estimulem condutas pró-sociais. Por outro lado, permitiu detectar que a nãoutilização de uma estratégia efetiva de recrutamento e retenção dos participantes pode comprometer a transformação deste projeto em um programa.
\end{abstract}

Palavras-chave: programas preventivos, habilidades sociais, avaliação de programas.

\section{PREVENTIVE PROGRAMS EVALUATION: EXPERIENCE STORY}

BSTRACT. A critical analysis of a project allows refining it by identifying the aspects that make it difficult in order to facilitate the inclusion of effective strategies for the correction of the problems. Some authors suggest that the evaluation of preventive programs is made, considering the stages: analysis of the problem, delineation of the program, accomplishment of test-pilot, accomplishment of advanced test, dissemination or enlargement for other contexts. The objective of this paper is to evaluate a preventive project that aim to stimulate the development of social abilities in children from 6 to 8 years old. It was observed that the analyzed project has some positive aspects: it promotes subtle changes in the behavior of the children and sensitizes the parents in order to stimulate pro-social behaviors. On the other hand, it allowed to detect that a non use of an effective strategy of recruitment and retention of the participants can compromise the transformation of this project in a program.

Key words: Preventive programs, social abilities, evaluation of programs.

\section{EVALUACIÓN DE PROGRAMAS PREVENTIVOS: RELATO DE EXPERIENCIA}

RESUMEN A. El análisis riguroso de un proyecto permite refinarlo, identificando aspectos dificultadores del mismo y facilitando la inclusión de estrategias efectivas para corrección de los problemas. El presente artículo procuró evaluar un proyecto preventivo que busca estimular el desarrollo de habilidades sociales en niños de 6 a 8 años. El análisis fue hecho tomando por base las etapas: análisis del problema, delineamiento del programa, realización de test piloto, realización de test avanzado, diseminación o ampliación para otros contextos. Se constató que el proyecto analizado tiene varios aspectos positivos: promueve cambios sutiles en el comportamiento de los niños y sensibiliza a los padres y profesores para que estimulen conductas pre-sociales. Por otro lado, permitió detectar que la no utilización de una estrategia efectiva de reclutamiento y retención de los participantes puede comprometer la transformación de este proyecto en un programa.

Palabras-clave: programas preventivos, habilidades sociales, evaluación de programas..

* Doutora em Psicologia Clínica pela USP. Coordenadora e professora do curso de Psicologia do Centro Universitário Positivo (UNICENP).

\# Psicóloga. Pós-graduanda em Educação Especial da Universidade Federal de São Carlos.

Il Acadêmico do curso de Psicologia do Centro Universitário Positivo (UNICENP). 
A prevenção de problemas é uma das áreas de estudo e de atuação para a qual a psicologia cada vez mais tem se voltado. Conforme afirma Melo (2003), há duas razões fundamentais para embasar a existência de trabalhos preventivos: resultados mais positivos em longo prazo e menores custos que a remediação. Esses fatores elevam o interesse do Estado e da sociedade em financiá-los. Para ações preventivas, faz-se necessário delimitar fatores desencadeantes de problemas futuros e a intervenção possível em tais fatores. De acordo com Zamberlan, Freitas, Fukahori e Novais (2003), para estruturar ações preventivas é necessário analisar e contextualizar os processos de mudança pelos quais um organismo passa, o que permite a identificação dos fatores de proteção e de risco associados a diversos comportamentos.

Conforme ressaltam Najaka, Gottfredson e Wilson (2001), para a montagem de programas preventivos adequados é importante identificar as variáveis que levam a problemas comportamentais, e práticas preventivas para evitar tais problemas demonstraram bons resultados. Os autores citam estudos que analisam programas de prevenção de abusos de substâncias, de violência, comportamento anti-social, de saúde mental e de desenvolvimento infantil.

Melo (2003) constatou que, no Brasil, implantar programas de atendimento psicológico de caráter preventivo é uma atitude bastante inovadora, visto que a literatura brasileira sobre prevenção ainda é escassa.

O estudo dos aspectos que caracterizam um programa pode constituir-se como forma de análise desse programa. Para Nelson, Westhues e MacLeod (2003), aspectos como análise da etapa de desenvolvimento dos participantes, duração e custo do programa, a teoria de seu embasamento e o tempo de validade podem contribuir na avaliação de programas preventivos.

Dumka, Roosa, Michaels e Suh (1995) propõem que se divida a avaliação de programas preventivos em cinco fases: análise de problema, delineamento do programa, teste-piloto, teste avançado e disseminação do programa. É na fase de análise do problema que se definem e se identificam os fatores de risco e de proteção, o que o autor considera ser fundamental para uma maior efetividade dos programas preventivos. Nesta fase, Dumka e cols. (1995) incluem também a descrição da problemática-alvo, identificação do grupo de risco e a avaliação da acessibilidade a este grupo. Na segunda fase ocorre o delineamento do programa, que se inicia com consulta ao público-alvo quanto aos seus sistemas de valores e crenças. Faz-se então a seleção dos objetivos, a descrição dos comportamentos a serem modificados, a escolha dos instrumentos de avaliação (estes devem estar em consonância com os objetivos), a escolha dos métodos de mudança, a decisão sobre a extensão do programa (definindo se o público-alvo será universal ou específico), a quantidade de objetivos a serem alcançados, a duração do programa e a freqüência das atividades, o planejamento do recrutamento dos participantes, a forma de retenção deles no programa $\mathrm{e}$, finalmente, o planejamento dos procedimentos do programa (o conteúdo a ser trabalhado, os materiais a serem utilizados, número de sessões, o horário dos encontros, o meio de transporte dos participantes do programa, entre outros). Após a delimitação do programa passa-se para a terceira fase descrita por Dumka e cols. (1995), em que é realizado o testepiloto, o qual permite avaliar o delineamento do programa e realizar as modificações necessárias. Nessa fase a avaliação incide prioritariamente nos aspectos de recrutamento e retenção, procedimentos utilizados e opinião dos participantes e condutores acerca do programa. Após os ajustes necessários, passa-se para a fase de teste avançado, que tem como objetivo estabelecer se o programa experimental alcançou os objetivos descritos na fase de delineamento. A última fase de avaliação de programas, denominada disseminação, analisa se o programa foi ampliado para outros contextos e outras populações. É importante salientar que essas fases não são lineares e se relacionam constantemente.

A disseminação dos programas para novos contextos é um passo relevante, uma vez que provoca o aumento do número de pessoas a serem beneficiadas. Rotheram-Borus e Duan (2003) descrevem quatro barreiras para a realização dessa fase. Uma seria a centralização do programa no pesquisador principal, dependendo apenas da persistência e comprometimento deste e do seu interesse pela disseminação. A segunda barreira diz respeito à carência de teoria de embasamento, o que pode ser decorrente do baixo número de estudos do processo de mudança comportamental. A terceira dificuldade se refere à focalização do programa em mudança comportamental, esquecendo-se de trabalhar a aderência a ele até o seu término. A última barreira seria a implantação do programa com fidelidade ao longo do tempo, desconsiderando-se as particularidades dos diferentes indivíduos participantes e do local implantado, como também não permitindo que se conheçam quais são os componentes essenciais do programa, já que não se fazem testes alternando os diferentes componentes. 
Para que haja equilíbrio entre a fidelidade ao programa original e sua adequação às necessidades dos participantes ou do local de implantação, Castro, Barrera e Martinez (2004) sugerem que, antes de implantar um programa preventivo em um contexto diferente do original, seja realizado um planejamento prévio no qual se avaliem as características do público-alvo, os profissionais que aplicarão o programa e a comunidade à qual o público-alvo pertence (rural, urbana, latina, norte-americana, indígena, etc.). Os autores sugerem três dimensões a respeitar: a cognitivo-informacional (características lingüísticas e estágio do desenvolvimento dos participantes); a afetivo-motivacional (como gênero, origem étnica e status sócio-econômico); e a ambiental (aspectos da comunidade local e infra-estrutura).

Para que ocorra a disseminação é necessário tornar o programa comercializável, isto é, interessante para a comunidade não científica. Alguns fatores a observar são: pensar em um nome atrativo e de fácil entendimento para o programa; criar caminhos, palestras e anúncios, para divulgá-lo ao público-alvo; conseguir financiamentos governamentais, de ONGS ou de empresas privadas. Segundo Rotheram-Borus e Duan (2003), se pesquisadores de universidades adotassem uma orientação mercadológica para disseminar e refinar as intervenções deles por meio de um modelo privado, ocorreria uma mudança de paradigma e esta poderia acelerar a disseminação de intervenções eficazes em nível mundial.

Outros fatores apontados por Rotheram-Borus e Duan (2003) que contribuem para a ampliação bemsucedida de programas são: formação de profissionais capazes de conduzir o programa, criação de uma rede de comunicação entre os diferentes ambientes em que o programa será implantado e constante avaliação desse programa nos diversos lugares.

Greenberg (2004) ressalta que, na avaliação dos programas, deve-se considerar se estes estão integrados por três dimensões: etapas/ano escolar, nível de intervenção e instituições comunitárias. A primeira dimensão de integração pressupõe que os programas preventivos sejam sequienciais e ocorram durante toda uma etapa de vida, geralmente a escolar. Ações para viabilizar tal orientação implicam que pesquisadores e profissionais condutores dos programas desenvolvam conjuntamente um planejamento escolar de longo prazo, assegurem uma infra-estrutura adequada durante todo o programa e façam avaliações do processo e dos resultados da integração. A segunda dimensão propõe a integração de programas com diferentes formas de seleção do público-alvo. Assim, a mesma pessoa pode participar de um programa com público-alvo universal e de outro com população mais específica, como a de um grupo de risco, por exemplo A terceira dimensão considerada por Greenberg (2004) enfatiza a obtenção de informações sobre o indivíduo participante do programa em todas as instituições que lhe prestam atendimento (escolas, agências comunitárias, agências de serviço à família, de saúde mental e hospitais).

Para Alvarez (1992), a comunicação dinâmica na coleta de informações (professores, médicos, assistentes sociais) e de diferentes instituições (educação, saúde, serviço social) é uma estratégia que permite avaliar os efeitos de um programa preventivo.

Nelson e cols. (2003) enfatizam a origem racial e o status socioeconômico dos selecionados como variáveis que alteram o sucesso dos programas norteamericanos. Os autores alertam para as condições antes da intervenção, citando que quanto piores são as condições pré-intervenção do grupo-alvo, maior é a diferença entre pré e pós-programa. Quanto aos componentes envolvidos, Nelson e cols. (2003) enfatizam a quantidade de componentes neles existente. Enquanto alguns estão voltados exclusivamente para crianças, existem outros que priorizam os pais e/ou famílias destas. Melhores resultados são obtidos com programas que tenham múltiplos componentes (orientação a pais, orientação a professores, reforço acadêmico, atividades esportivas e culturais, etc.); porém a inclusão de vários componentes torna mais complexa a implementação do programa, exige maior número de pessoas envolvidas e dificulta a constatação de qual componente é mais efetivo. Programas com múltiplos componentes têm mais chances de atingir os resultados, mas não permitem análise dos resultados associados a cada componente.

August, Egan, Realmuto e Hektner (2003) citam pesquisas de como determinados componentes funcionam diferentemente para cada subgrupo de indivíduos e como um componente pode ser mais importante que outro. Ialongo, Poduska, Werthamer e Kellam (2001), em suas pesquisas, compararam duas intervenções preventivas, sendo que uma delas era voltada para o manejo de professores pré-escolares e outra tinha como alvo melhorar a comunicação paisescola e ensinar práticas parentais adequadas. Segundo Ialongo e cols. (2001), a intervenção no comportamento dos professores foi mais efetiva do que a dirigida ao comportamento dos pais, no que se refere à redução de problemas de conduta e de necessidade de serviço psicológico pós-programa das crianças até a idade dos 12 anos. August e cols. (2003) conjecturam que, se fossem oferecidos diversos 
componentes de um mesmo programa para que as famílias escolhessem de quais gostariam de participar, é muito provável que elas optassem pelos componentes que acreditam ser compatíveis com suas necessidades, interesses e conveniências diárias, o que poderia aumentar a participação das famílias nos programas, fator este que constitui dificuldade em muitos programas.

$\mathrm{Na}$ avaliação dos programas quanto à teoria que os fundamenta, Rotheram-Borus e Duan (2003) destacam as intervenções como um importante aspecto a ser avaliado. Essencialmente, todas as ações eficazes são embasadas teoricamente e reconhecem o processo pelo qual as mudanças ocorrem; no entanto, estabelecer claras relações entre os procedimentos do condutor do programa e a prevenção de problemas tem sido uma questão tumultuada e de difícil acesso.

A manutenção do engajamento nos programas é outra dificuldade. Nelson e cols. (2003) defendem que, para se assegurar a assiduidade ao programa, este deve ser de curta duração e de baixo custo. De acordo com a pesquisa dos autores, os programas preventivos para crianças pré-escolares, de maior duração e intensidade (de no mínimo um ano), alcançam médios efeitos e estes permanecem em longo prazo, enquanto programas para pré-escolares, de menor duração e menos intensos, conseguem somente efeitos pequenos e de curto prazo. Assim, diante do impasse entre oferecer programas breves e de baixo custo para manter o engajamento dos pais e oferecer programas de maior duração para obter melhores resultados, parece que o ideal é buscar o equilíbrio entre duração do programa, custo e efeitos alcançados. Assim, o período de duração e a freqüência das atividades são variáveis importantes para se determinar a qualidade de um programa e devem ser bem escolhidas. $\mathrm{O}$ efeito de um programa preventivo subentende provocar mudanças em padrões de comportamento potencialmente prejudiciais e mantê-las no tempo. De nada adianta propor um programa preventivo que seja de baixo custo e pequena duração, mas cujos ganhos rapidamente são perdidos. Rotheram-Borus e Duan (2003) mostram que a maioria das intervenções se preocupa mais em provocar alterações iniciais do que em preservar ao longo do tempo os comportamentos adequados adquiridos no programa. Para Silveira, Silvares e Marton (2003), a realização de follow-up pode ser uma forma de propiciar acompanhamento do programa, avaliando os ganhos em curto, médio e longo prazos.

Pentz (2004) reflete sobre a utilização do programa em diferentes momentos e questiona se existe uma "data de validade" para os programas preventivos. A autora diz que a maioria dos programas preventivos registrados oficialmente nos Estados Unidos foi testada somente nos anos 80 e 90, porém características importantes do público-alvo mudaram, não se assegurando que atualmente os programas ainda sejam eficazes, o que justifica a necessidade de avaliação dos programas existentes.

Os condutores dos programas preventivos constituem outra variável relevante. Anteriormente eram percebidos como os responsáveis pela promoção da prevenção nas comunidades, mas com o passar dos anos aumentou o envolvimento de líderes comunitários com o tema, ampliando-se as possibilidades de disseminação das ações.

Pela revisão apresentada percebe-se que muitos aspectos dos programas preventivos são objeto de estudo, porém, apesar dos muitos programas implantados e disseminados, o tema ainda é uma novidade e suscita muitas questões para investigação.

Apresentaremos a análise e avaliação de um projeto preventivo. A proposta de análise de Dumka e cols. (1995) constitui o instrumento central de discussão, não se negligenciando as observações de outros autores mencionados na revisão bibliográfica.

\section{AVALIAÇÃO DO PROJETO “GRUPO DE CRIANÇAS: DESENVOLVENDO HABILIDADES SOCIAIS NA INFÂNCIA".}

O projeto avaliado está em desenvolvimento desde 2003, na clínica-escola de um curso de Psicologia de Curitiba, e tem como pressuposto a promoção de habilidades sociais na infância como uma maneira de prevenir comportamentos desajustados. De acordo com Del Prette e Del Prette (2005), a expressão "habilidades sociais" aplica-se às diferentes classes de comportamentos sociais do repertório de um indivíduo, como empatia, resolução de problemas, assertividade e respeito aos direitos humanos. Estas contribuem para um relacionamento saudável e produtivo com as pessoas. "O desempenho competente das habilidades sociais tem alta probabilidade de obter conseqüências reforçadoras no ambiente social" (Del Prette \& Del Prette, 2005, p. 31).

O presente estudo escolheu como base de análise as cinco fases propostas por Dumka e cols. (1995), as quais são constantemente inter-relacionadas: análise do problema, delineamento do projeto, teste-piloto, teste avançado e ampliação do programa. Um ponto relevante a ser destacado é que se estará analisando um projeto que no futuro pode a vir a ser um programa, enquanto a proposta de Dumka e cols. 
(1995) visa à avaliação de programas. Não foram encontrados instrumentos específicos para a avaliação de projetos. Além disso, é importante ressaltar que o projeto foi desenvolvido sem os idealizadores terem conhecimento das etapas propostas pelos autores, o que fez com que as etapas de teste-piloto e teste avançado fossem concomitantes opondo-se ao que o autor sugere. Contudo, tal diferença não impede a análise do projeto pelas etapas.

Um projeto diferencia-se de um programa por envolver um número menor de participantes. Pode-se desenvolver uma nova sistemática de manejo profissional em um projeto e, quando tal sistemática está bem-estruturada, têm-se elementos para propor um programa, disseminando aquela perspectiva e permitindo que a população em geral possa usufruir dela. Assim, parece-nos cabível utilizar os critérios de avaliação de programas na análise de um projeto.

A figura abaixo descreve a evolução do Projeto "Grupo de crianças: desenvolvendo habilidades sociais na infância, seguindo as etapas propostas por Dumka e cols. (1995). Tais etapas serão discutidas o longo do texto.
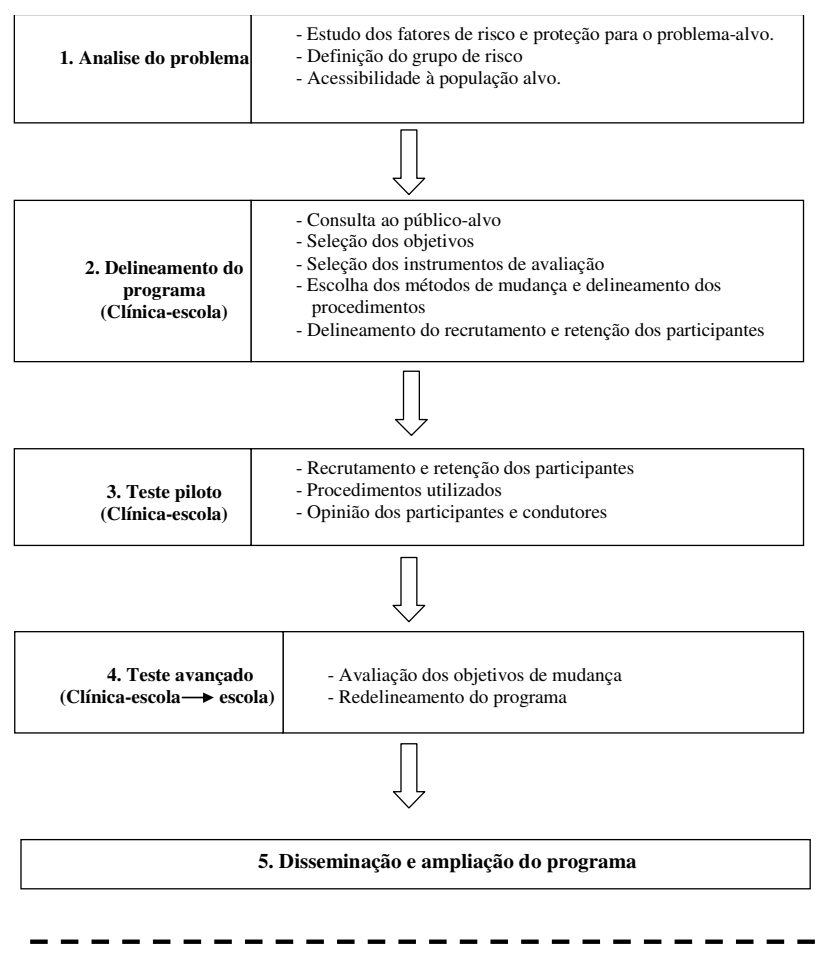

Figura 1. Esquema de Avaliação de um Projeto de Desenvolvimento Habilidades Sociais na Infância.

\section{Análise do problema}

Em relação ao estudo dos fatores de risco e proteção para a problemática-alvo, vários pesquisadores investigaram estes fatores na aquisição e manutenção de comportamentos pró-sociais (Brotman e cols., 2003). Além de fatores sociais, os estudos destacaram aspectos biológicos - tais como predisposição genética, disfunções neurológicas e problemas motores - como correlacionados com o comportamento anti-social (Bermann, 1997; Brennan \& Raine, 1997).

No Brasil, Silveira e cols. (2003) apontaram alguns fatores que contribuem para a aquisição de comportamentos não habilidosos das crianças, destacando: atrasos cognitivos, déficit em autocontrole, habilidades sociais parentais deficitárias, comportamentos parentais hostis, baixa renda, baixa escolaridade, entre outros. Segundo esses autores, o desenvolvimento de condutas parentais adequadas e intervenções em contextos mais amplos, ligados a políticas públicas, são considerados fatores de proteção que fomentam um repertório comportamental adequado.

Outras variáveis que interferem na competência social extrapolam os objetivos do projeto em análise, sendo que este atuou somente em algumas das variáveis que poderiam constituir fatores de risco ao relacionamento interpessoal, como déficit de autocontrole da criança, habilidades sociais parentais deficitárias, comportamentos parentais hostis, má discriminação de contingências por parte da criança e de seus pais. Os fatores de proteção são empatia, afetividade, habilidades comunicativas e assertividade.

Com relação à definição do grupo de risco, como o projeto se caracteriza pela prevenção, o grupo-alvo escolhido não era de crianças com comportamentos de risco no que se refere às habilidades sociais. $O$ público-alvo foram crianças de 6 a 8 anos, as quais foram selecionadas por se encontrarem no início da escolarização e estarem inseridas nos primeiros contatos sociais extrafamiliares.

Segundo Lacourse e cols. (2002) e Caspi, Jaffe, Moffitt e Taylor (2004), este momento, o da primeira participação em grupos extrafamiliares, é essencial para uma boa adaptabilidade social e acadêmica futura.

Quanto à acessibilidade à população-alvo, houve facilidade em contactar as pessoas para participarem do projeto, uma vez que estas residiam nas proximidades da clinica-escola onde o trabalho seria desenvolvido.

\section{Delineamento do programa}

O delineamento do programa pressupõe algumas tarefas: consulta ao público-alvo, seleção dos objetivos e dos instrumentos de avaliação, escolha dos métodos de mudança, delineamento do recrutamento e 
retenção dos participantes, e delineamento dos procedimentos.

A consulta ao público-alvo foi feita por meio de reuniões que apresentavam os objetivos do projeto e informavam aos pais os temas propostos nos encontros.

$\mathrm{Na}$ seleção dos objetivos a meta principal foi estimular o engajamento das crianças em comportamentos habilidosos socialmente, tendo como objetivos específicos aumentar comportamentos prósociais, diminuir comportamentos anti-sociais e não assertivos e propiciar o desenvolvimento de estilo parental autoritativo.

A seleção dos objetivos teve como base as necessidades levantadas pelos pais e/ou responsáveis. A meta principal foi estimular o engajamento das crianças em comportamentos habilidosos socialmente, visando aumentar comportamentos pró-sociais, diminuir comportamentos anti-sociais e nãoassertivos, melhorar habilidades sociais parentais e propiciar o desenvolvimento de estilo parental autoritativo.

Quanto à seleção dos instrumentos de avaliação, foram utilizados o Children Behavior Check List (CBCL), a observação sistemática e a observação de atividades de interação das díades pais/crianças.

O CBCL é um inventário de avaliação dos comportamentos de crianças e adolescentes entre 4 e 18 anos que permite coletar informações dos pais sobre as competências, problemas emocionais e comportamentais das crianças, enfocando uma de suas vertentes de análise.

A observação sistemática do comportamento das crianças feita por registro de eventos permitiu avaliar a mudança dos comportamentos habilidosos, não habilidosos e agressivos das crianças no decorrer dos encontros.

A observação de atividades de interação das díades pais-criança, realizada também por registros de eventos, antes e após as intervenções, auxiliou no entendimento das relações entre pais e filhos.

As observações qualitativas assistemáticas referem-se às percepções dos condutores do programa em relação à mudança dos comportamentos das crianças no decorrer dos encontros.

Quanto à escolha dos métodos de mudança e delineamento dos procedimentos, definiu-se que o projeto seria desenvolvido em oito encontros de duração de uma hora e meia, com periodicidade semanal com crianças, e oito encontros também semanais de duração de uma hora e meia com os seus pais. Conforme afirmam Nelson e cols. (2003), a periodicidade e duração são variáveis importantes para se determinar a qualidade de um programa e devem ser bem escolhidas. Temas relacionados a habilidades sociais, trabalhados por meio das atividades lúdicas, constituem o cerne dos encontros com as crianças. Os terapeutas utilizam os princípios da análise do comportamento como teoria-base para as intervenções, fazendo uso de princípios como modelagem, reforçamento, extinção, sempre apoiados em uma análise funcional de cada interação. Para RotheramBorus e Duan (2003) definir o embasamento teórico de um programa é essencial.

Quanto à escolha dos métodos de mudança e delineamento dos procedimentos, definiu-se que o projeto seria desenvolvido em oito encontros com as crianças e oito encontros com os pais, com periodicidade semanal e duração de uma hora. Nesta perspectiva, conforme afirmam Nelson e cols. (2003), a periodicidade e duração são variáveis importantes para se determinar a qualidade de um programa.

Os terapeutas utilizam os princípios da análise do comportamento como teoria-base para as intervenções, fazendo uso de princípios como modelagem, reforçamento, extinção, sempre apoiados em uma análise funcional de cada interação. Para RotheramBorus e Duan (2003), definir o embasamento teórico é um aspecto importante na elaboração de programas.

Além disso, houve preocupação em equilibrar as ações dirigidas às mudanças comportamentais na área de habilidades sociais e outras que enfocavam especificamente aderência ao projeto, conforme orientam Rotheram-Borus e Duan (2003). Nos encontros com as crianças foram empregadas atividades lúdicas, tais como dramatizações com fantoches, leitura e reflexão sobre histórias referentes ao tema das habilidades sociais, jogos, dinâmicas e construções de desenhos em grupo. Nos encontros com os pais foram discutidos temas referentes ao desenvolvimento infantil, aos princípios da análise do comportamento, aos estilos parentais e habilidades sociais. Os pais expunham as suas dificuldades, descreviam minuciosamente os seus comportamentos em relação à criança e eram orientados na elaboração de análises funcionais, o que facilitou a emissão de comportamentos parentais habilidosos e a estimulação do comportamento pró-social da criança, questões relacionadas ao primeiro aspecto: mudança comportamental. Estratégias e dinâmicas motivadoras compõem o manejo dos encontros tanto das crianças quanto dos pais.

No que se refere ao delineamento do recrutamento e retenção dos participantes, o projeto foi divulgado entre as crianças participantes de atividades de esporte na instituição de ensino em cuja clínica psicológica o projeto seria desenvolvido. As crianças e seus pais 
receberam cartas-convite explicando em linhas gerais o projeto e convidando-os para uma reunião para mais esclarecimentos. Os critérios para escolha dos participantes foram a faixa etária em que as crianças se encontravam e a disponibilidade de horário para a participação nos encontros, tanto das crianças quanto de seus pais. Dos quarenta pais e crianças que receberam convite, seis pais vieram ao encontro e participaram do projeto proposto.

Ao analisar os fatores que contribuíram para a manutenção das crianças no projeto, percebeu-se que o horário de oferta da atividade e a sua duração eram fatores importantes. Adequou-se, então, o horário das sessões de forma que elas fossem realizadas após as atividades da educação física e de maneira que tivessem duração de no máximo uma hora.

A escuta atenta das dificuldades dos participantes, mesmo que estas não fossem o alvo da intervenção, constituiu uma estratégia de acolhimento dos condutores do projeto posteriormente relatada como significativa pelos pais para sua decisão de manter-se no projeto. Foram realizados contatos telefônicos quando os participantes faltavam aos encontros, estimulando-os a continuar no projeto, ao mesmo tempo em que se mostrava a importância da presença deles para o grupo. $\mathrm{O}$ fato de o projeto disponibilizar todos os materiais necessários para as atividades, evitando os gastos financeiros por parte dos freqüentadores, foi outro aspecto que favoreceu a adesão.

\section{Teste-piloto}

Quanto a recrutamento e retenção, percebeu-se que as estratégias adotadas não foram suficientemente efetivas. Conforme aponta Rotheram-Borus e Duan (2003), tornar os programas interessantes à comunidade não científica em seus mínimos detalhes é importantíssimo. Somente seis díades pais/crianças responderam às cartas-convite e destas, apenas quatro díades permaneceram até o término.

No recrutamento e retenção dos participantes percebeu-se que as estratégias adotadas não foram suficientemente efetivas, já que das quarenta díades pais/crianças recrutadas, somente seis responderam às cartas-convite, e destas, apenas quatro díades permaneceram até o término.

Os procedimentos utilizados atenderam aos objetivos propostos, além de despertarem o interesse das crianças. Em se tratando de projeto dirigido à prevenção, não se evidenciaram mudanças comportamentais significativas antes e após a intervenção.
No que se refere às opiniões dos participantes, o relato dos pais era de que as atividades estavam bem estruturadas e de fácil compreensão, sendo que observaram pequenas melhoras no comportamento dos filhos no que se refere ao manejo nas interações sociais.

Durante os encontros, os condutores das atividades perceberam que as crianças engajaram-se mais facilmente na interação com pares, mantendo menos interações aversivas.

\section{Teste avançado}

$\mathrm{O}$ teste avançado refere-se à avaliação dos objetivos de mudança, verificando se estes foram alcançados. Apesar de esta etapa ter ocorrido concomitantemente à etapa de teste-piloto no projeto em análise, percebe-se que o projeto provocou mudanças positivas nos comportamentos da criança e na interação com os pais. Verificou-se na análise das categorias de observação sistemática das sessões com as crianças que os comportamentos habilidosos socialmente aumentaram no decorrer dos encontros, principalmente na interação com adultos. Quanto aos escores obtidos no CBCL, antes e após os atendimentos, em média, a percepção dos pais indica alterações benéficas mínimas no índice de distúrbio externalizante e distúrbio total. Este aspecto foi discutido no grupo de pesquisa e percebeu-se que as mudanças não são intensas em virtude de tratar-se de uma intervenção preventiva, não havendo problemas previamente citados. Julgou-se, porém, necessário rever o conteúdo das sessões de crianças, assim como as estratégias utilizadas nas sessões, buscando alternativas que viabilizassem mudanças mais observáveis no comportamento.

No redelineamento do programa optou-se por desenvolver o projeto numa escola pública da região de Curitiba, o que favorece o acesso a maior número de pessoas. Dada a natureza preventiva do projeto, limitou-se o público-alvo a crianças de uma classe de pré-escola, aos pais e à professora responsável pela classe. A definição da turma de pré-escola em que a atividade seria realizada foi feita pela diretora da escola. Os pais foram convidados a participar do projeto por intermédio de cartas-convite anexadas à agenda das crianças.

Os encontros aconteciam uma vez por semana no final da tarde, logo após o término das aulas das crianças. Mantiveram-se os objetivos relativos a favorecer a aquisição dos comportamentos habilidosos pelas crianças. A atuação envolvendo múltiplos componentes (grupo com as crianças, grupo de orientação aos pais e grupo de orientação com as 
professoras) foi mantida, incluindo-se o componente orientação de professores no programa. De acordo com Nelson e cols. (2003), melhores resultados são obtidos com programas que têm múltiplos componentes, porém estes são de implementação mais complexa. O objetivo da inclusão da orientação a professores era instrumentalizar a professora para que, durante as aulas, continuasse estimulando as habilidades sociais que as crianças desenvolviam nas sessões, ação esta que se mostrou efetiva, já que a professora passou a incluir em suas aulas atividades que estimulavam comportamentos pró-sociais das crianças.

Além dos instrumentos empregados no testepiloto, a inclusão da orientação aos professores levou à necessidade de acrescentar um registro de observação sistemática das crianças em ambiente de classe nas aulas regulares, podendo-se assim analisar se havia ou não variação dos comportamentos delas em diferentes ambientes, bem como avaliar o grau em que se estabelecia a generalização para o contexto escolar dos comportamentos aprendidos nas sessões.

Após o redelineamento, foi realizado um segundo teste-piloto. O recrutamento não atingiu um bom resultado. Apenas seis crianças de uma classe de 30 alunos participaram do projeto, o que representa $20 \%$ dos alunos. Dessas seis crianças, apenas duas participaram até o término do projeto, dando uma adesão de 33,3\%. Esperava-se que a realização na escola aumentasse a adesão, o que não aconteceu. É possível que, em se restringindo a uma turma da escola, a margem de pais com disponibilidade de tempo e interesse seja menor. Quando o projeto era divulgado em um projeto de extensão (de atividades esportivas) de uma instituição de ensino, os pais lá presentes já eram pais mobilizados a buscar atividades extracurriculares na educação dos filhos. Quando investigados os motivos para a desistência dos participantes, estes referiram circunstâncias que impossibilitaram a participação, como trabalho no turno no qual o projeto ocorria e mudança da criança de escola e de local de residência.

As crianças que participaram até o término do projeto demonstraram avanços no manejo interpessoal e os pais relataram ganhos com a participação no projeto. Sendo o objetivo da $4^{\mathrm{a}}$ etapa proposta por Dumka e cols. (1995) avaliar se os objetivos de mudança foram alcançados, pode-se dizer que os pontos positivos do projeto se mantiveram, mas as novas estratégias (mudança do local dos encontros para a escola, sessões ao final dos horários das aulas, divulgação via caderneta dos alunos, envolver uma única turma) não atingiram as metas esperadas. Houve elevação do índice de comportamentos habilidosos socialmente emitidos pelas crianças no decorrer dos encontros, principalmente na interação com adultos. Os escores obtidos no CBCL, antes e após os atendimentos, indicam leves alterações positivas no índice de distúrbio externalizante e distúrbio total. Em se tratando de um projeto preventivo, é grande a importância destas pequenas mudanças para o repertório da criança.

\section{Disseminação ou ampliação do programa}

Esse aspecto refere-se à última fase proposta por Dumka e cols. (1995), na qual o programa é ampliado para outros contextos e outras populações. Essa fase não foi iniciada. Pretende-se ajustar o projeto e testar as alterações propostas, para no futuro poder aplicá-lo em outras populações e contextos, transformando-o então em um programa. Quando o projeto estiver suficientemente estruturado e pronto para disseminação em forma de programa, é interessante analisar as barreiras descritas por Rotheram-Borus e Duan (2003); ou seja, ele não poderá centralizar-se em um único pesquisador; necessitará de bom embasamento teórico; precisará focalizar mudança comportamental, mas não poderá negligenciar o aspecto de aderência ao programa; deverá fornecer mecanismos de ajuste que atendam às potencialidades dos participantes ou do local em que será implementado.

\section{CONSIDERAÇÕES FINAIS}

No campo das habilidades sociais realiza-se uma quantidade cada vez maior de estudos, sugerindo a importância desse tema na atualidade. O presente projeto mostrou-se eficaz ao modelar condutas prósociais, muito embora apresente dificuldades metodológicas e de implementação. Vale ressaltar que estas dificuldades não ocorrem de maneira isolada, ao contrário, a mensuração dos dados ainda é um desafio a ser superado dentro do campo científico, assim como a implementação de projetos requer freqüentes ajustes, para que eles se ajustem às necessidades do mundo real. A avaliação cautelosa e apoiada em critérios de análise claros, como os descritos por Dumka e cols. (1995), fornece maior consistência aos projetos, fazendo com que programas futuros tenham maior chance de êxito.

Espera-se que este trabalho possa encorajar futuras publicações que auxiliem na busca de ajustamentos e permitam uma melhora na qualidade de implantação de programas preventivos. 


\section{REFERÊNCIAS}

Alvarez, E. C. (1992). Tareas del psicólogo en promoción y prevención de la salud en atención primaria. Papeles del psicólogo, 53. Recuperado em 07 de outubro de 2005, de http://www.cop.es/papeles/vernumero.asp?ID=537.

August, G. J., Egan, E., Realmuto, G. M. \& Hektner, J. M. (2003). Parceling component effects of a multifaceted prevention program for disruptive elementary school children. Journal of abnormal child psychology, 31, 515-527.

Bermann, M. E. (1997). Biopsychosocial approaches to understanding human aggression: The first 30 years. Clinical Psychology Review, 17, 585-588.

Brennan, P. A. \& Raine, A. (1997). Biosocial bases of antisocial behavior: Psychophysiological, neurological and cognitive factors. Clinical Psychology Review, 17, 589-604.

Brotman, L. M., Klein, R. G., Kamboukos, D., Brown, E. J., Coard, S. I. \& Sosinsky, L. S. (2003). Preventive intervention for urban, low-income prechoolers at familial risk conduct problems: A randomized pilot study. Journal Clinical Child Adolescent Psychology, 32(2), 246-257.

Caspi, A., Jaffee, S. R., Moffitt, T. E. \& Taylor, A. (2004). Physical maltreatment victim to antisocial child: Evidence of an environmentally mediated process. Journal Abnourmal Psychology, 1, 44-55.

Castro, F. G., Barrera, M. \& Martinez, C. R. (2004). The cultural adaptation of prevention interventions: Resolving tensions between fidelity and fit. Prevention Science, 5(1), 41-45.

Del Prette, Z. A. P. \& Del Prette, A. (2005). Psicología das habilidades sociais na infância: teoria e prática. Petrópolis: Vozes.

Dumka, L. E., Roosa, M. W., Michaels, M. L. \& Suh, K. W. (1995). Using research and theory to develop prevention programs for high-risk families. Family Relations, 44, 78-86.

Greenberg, M. T. (2004). Current and future challenges in schoolbased prevention: The researcher perspective. Prevention Science, 5(1), 5-13.

Ialongo, N., Poduska, J., Werthamer, L. \& Kellam, S. (2001). The distal impact of two first grade preventive interventions on conduct problems and disorder and mental health service need and utilization in early adolescence. Journal of Emotional and Behavioral Disorders, 9, 146-160.

Lacourse, E., Côté, S., Nagin, D. S., Brendgen, M., Vitaro, F. \& Tremblay, R. E (2002). A longitudinal-experimental approach to testing theories of antisocial behavior development. Development and Psychopathology, 14(4), 909-924.

Melo, M. H. S. (2003). Uma intervenção multifocal na comunidade. Tese de Doutorado Não-Publicada. Programa de PósGraduação em Psicologia Clínica, Universidade de São Paulo, São Paulo.

Melo, M. H. S. \& Silvares, E. F. M. (2000). A Psicologia clínica e os programas preventivos de intervenção comunitária. Boletim de Psicologia, 1(113), 85-97.

Najaka, S. S., Gottfredson, D. C. \& Wilson, D. B. (2001). A metaanalityc inquiry into the relationship between selected risk factors and problem behavior. Prevention Science, 4(2), 257271.

Nelson, G., Westhues, A. \& MacLeod, J. (2003). A meta-analysis of longitudinal research on preschool prevention programs for children. Prevention \& Treatment, 6(1). Recuperado em 14 de outubro de 2005, de http://journals.apa.org/prevention/ volume6/pre0060031a.html.

Pentz, M. A. (2004). Form follows function: Designs for prevention effectiveness and diffusion research. Prevention Science, 5(1), 23-29.

Rotheram-Borus, M. J. \& Duan, N. (2003). Next generation of preventive interventions. Journal of the American Academy of Child \& Adolescent Psychiatry, 42, 518-526.

Silveira, J. M., Silvares, E. F. M. \& Marton, S. A. (2003). Programas preventivos de comportamentos anti-sociais: dificuldades na pesquisa e na implementação. Estudos de Psicologia, 20(3), 59-67.

Zamberlan, M. A. T., Freitas, M. G., Fukahori, L. \& Novais, D. S. (2003). Relações familiares e adolescência. Em M. A. T. Zamberlan (Org.), Psicologia e prevenção: modelos de intervenção na infância e na adolescência (pp. 29-63). Londrina: UEL.

Recebido em 24/01/2006 Aceito em 17/11/2006

Endereço para correspondência: Suzane Schmidlin Löhr. Centro Universitário Positivo. Núcleo de Ciências Biológicas e Saúde. Centro Psicológico. Rua Prof. Pedro Viriato Parigot de Souza, no 5300, Campo Comprido, CEP 81280-330, Curitiba-Pr. E-mail: lohr@ superig.com.br 